\title{
Application of ultraviolet light (UV) in dental medicine
}

\author{
Vladimir Panov, Tsvetelina Borisova-Papancheva.
}

Department of Conservative Dentistry and Oral Pathology, Faculty of Dental Medicine, Medical University - Varna, Bulgaria;

\begin{abstract}
Ultraviolet (UV) light is electromagnetic radiation with a wavelength shorter than that of visible light, but longer than X-rays, that is, in the range between $400 \mathrm{~nm}$ and $100 \mathrm{~nm}$. Because of its ability to cause chemical reactions and excite fluorescence in materials, ultraviolet light has a huge number of useful applications in modern medicine, for diagnostic and therapeutic purposes. UV rays are the oldest method humans have used for decontamination; it is used for inactivating viruses, bacteria and fungi.

In dental medicine, the diagnostic uses are concerned with the fluorescence of teeth with radiation exposures to small areas and doses no more than $5 \mathrm{~J} / \mathrm{cm} 2$. A number of dental procedures involve the use of Ultraviolet radiation in the oral cavity.
\end{abstract}

Keywords: Ultraviolet (UV) light, dental medicine

\section{Introduction}

Ultraviolet (UV) radiation is defined as a portion of the electromagnetic spectrum between $\mathrm{x}$-rays and visible light. The UV spectrum is divided into Vacuum UV (40-190 nm), Far UV (190-220 nm), UVC (220$290 \mathrm{~nm})$, UVB (290-320), and UVA (320-400 nm). The sun is a primary natural source of UV radiation. Artificial sources include curing lamps, tanning booths, germicidal lamps, black lights, halogen lights, mercury vapor lamps, high-intensity discharge lamps, fluorescent and incandescent sources, and some types of lasers (nitrogen lasers, excimer lasers, third harmonic Nd:YAG lasers).

Some UV exposure is essential for good health. It stimulates vitamin D production in the body. In medical practice, UV lamps are used for treating psoriasis and for treating jaundice in new born babies. UV radiation can also be used as phototherapy to suppress pathogenic cutaneous immune responses. UV radiation induces chemical reactions which are essential for the formation of pre-vitamin D3 from 7-

dehydrocholesterol. Keratinocytes and other cells produce different types of cytokines, such as IL-1 $\beta$ and 
TNF- $\alpha$ induced by ultraviolet radiation. UV inactivates the T-lymphocytes and antigen-presenting cells, which leads to inhabitation of the proliferative responses of lymphoid cells to mitogens and alloantigens.

Acute effects of UVR include erythema (skin redness) and tanning. Delayed effects include skin cancer induction and skin aging. A section on sunbeds is included that discusses their medical and cosmetic use and the associated hazards. Other less common uses of UVR in medicine such as photopheresis are mentioned and the article concludes with information on UVR hazard assessment and protection. UV radiation is the main factor responsible for skin cancers, including basal cell carcinoma, squamous cell carcinoma and possibly melanoma.

Although ultraviolet light is usually invisible, under some conditions children and young adults can see ultraviolet light down to wavelengths of about $310 \mathrm{~nm}$. Because of its ability to cause chemical reactions and excite fluorescence in materials, ultraviolet light has a huge number of useful applications in modern medicine (Table 1).

Table 1. UV applications in medicine

\begin{tabular}{|l|l|}
\hline wavelength & Applications \\
\hline $230-400 \mathrm{~nm}:$ & Optical sensors, various instrumentation \\
\hline $240-280 \mathrm{~nm}:$ & $\begin{array}{l}\text { Disinfection, decontamination of surfaces and water (DNA absorption } \\
\text { has a peak at 260 nm) }\end{array}$ \\
\hline $200-400 \mathrm{~nm}:$ & Forensic analysis, drug detection \\
\hline $270-360 \mathrm{~nm}:$ & Protein analysis, DNA sequencing, drug discovery \\
\hline $280-400 \mathrm{~nm}:$ & Medical imaging of cells \\
\hline $300-320 \mathrm{~nm}:$ & $\begin{array}{l}\text { Light therapy in medicine, effective long-term treatment for many skin } \\
\text { conditions like psoriasis, vitiligo, eczema }\end{array}$ \\
\hline $300-365 \mathrm{~nm}:$ & Curing of polymers \\
\hline
\end{tabular}

\section{Aim}

The aim of this study is to find different application of Ultraviolet (UV) light in dental medicine.

\section{Results}

- It may be difficult to recognize composite resin restorations that are correctly shade-matched and wellplaced by visual and tactile inspection, which can make the replacement of an existing resin restoration challenging. Many composite resins fluoresce under UV LED light, which can help dentists to detect resin material. The optimal excitation wavelength is $385-395 \mathrm{~nm}$. There are three types of resin: highly fluorescent, moderately fluorescent, and weakly fluorescent. In each instance, the UV light revealed the presence of resin after all resin was believed to have been removed. The use of UV illumination can be a useful technique for determining whether composite resin has been removed completely (1).

- Optical measurements were obtained for the determination of the wavelength emission and intensity for 15 modern resins using a spectrophotometer. Resin brands fluoresce at different wavelengths and with varying intensities. The practical use and comparison of the flashlights revealed that the most useful excitation wavelengths for resin detection were in the UVA range (365 and $380 \mathrm{~nm}$ ). Porcelain 
restorations and composite resin fillings exhibited different responses to these two wavelengths and thus use of both is recommended for forensic dental inspection (2).

- A study was designed to test the application of ultraviolet light to root canal walls, as a means of complementary immediate disinfection after the use of sodium hypochlorite. Root canals were infected ex vivo by Metzger Z. in 2007 with Enterococcus faecalis for 48 hours. Non-attached bacteria were washed away, and the remaining attached bacteria were subjected to disinfection, with $5 \%$ sodium hypochlorite alone or followed by exposure to ultraviolet light $(254 \mathrm{~nm}, 300 \mathrm{~mJ} / \mathrm{cm} 2)$. Root canals were then tested for remaining viable bacteria. Canals were obturated and tested again after 14 days. Sodium hypochlorite alone achieved negative cultures in only $47 \%$ of the cases, but $96 \%$ was achieved with sodium hypochlorite followed by ultraviolet light. This status was also maintained after 14 days. Illumination of root canals with ultraviolet light may be an effective supplementary means to achieve immediate disinfection of infected root canals (3).

- Bacteria of dental caries produce metabolic products (porphyrins) as part of their normal life cycle. Upon irradiation with ultraviolet light, these porphyrins fluoresce in a typical bright red color. „Caries Detector" provides local, selective illumination with concentrated ultraviolet light only of the study area. There aren't glares, which significantly increases the resolution of visual perception. Red fluorescent porphyries can be clearly seen, if available (4).

- There are many methods for the disinfection of impression material; but these conventional strategies present several disadvantages. Mohammad T. et al introduced in 2013 the UV and blue light as effective savable methods for the disinfection of the impression (alginate and silicon). The culture showed that all the silicone impressions gave a positive result after treatment with blue light and UV light except for a group that was treated with UV light for 20 minutes, which showed significant reduction in the bacterial growth. All the alginate impressions give positive culture after treatment with UV and blue light after 10 and 20 minutes. The silicon impression can be sterilized with UV light when treated for 20 minutes (5).

- In another study of Larsen T. et al in 2000, alginate, in addition to silicone rubber and red wax contaminated by Streptococcus salivarius, Fusobacterium nucleatum and five other bacteria in different suspension media were radiated for up to $18 \mathrm{~min}$, and the number of colony forming units was compared to non-radiated controls. The effect of ultraviolet radiation differed among bacterial species and depended on the organic content in the suspension. Generally, the bacterial reduction after ultraviolet radiation was below 4 log steps and thus insufficient for disinfection of dental impressions (6, 7).

- Dental restorations were examined in 2004 by A. Gatti in order to investigate their color stability. The change in color was achieved by an accelerated aging test with ultraviolet rays and evaluated, for each material, with the measurement of reflectance. Such a measurement was obtained by means of a video display computer working as a computerized photometer. The results show two different types of behavior: some materials turn slightly yellow, while others bleach (8).

- Ultraviolet radiation is used to control airborne microorganisms and environmental surface decontamination. It will destroy and inactivate airborne microorganisms (Table 2) on exposed surfaces 
or in the presence of products of unstable composition that cannot be treated by conventional means. UV radiation appears to be an effective disinfection method for some surgical tools. However, using this method is not recommended for tools that have overlapping parts, such as needle holders, or other structures that cannot be exposed directly to UV radiation.

Table 2. Inactivation of airborne microorganisms - \% destruction and dose

\begin{tabular}{|l|l|l|l|}
\hline Microorganisms & dose Ws/m & \\
\hline Desired \% destruction & $50 \%$ & $90 \%$ & $99.9 \%$ \\
\hline S.enteritidis & 12.1 & 40 & 120 \\
\hline Escherichia coli & 9 & 30 & 90 \\
\hline $\begin{array}{l}\text { Micrococcus } \\
\text { sphaeroides }\end{array}$ & 30 & 100 & 300 \\
\hline Streptococcus viridans & 6 & 20 & 60 \\
\hline Saccharomyces spp. & 24 & 80 & 240 \\
\hline Aspergillus glaucus & 180 & 600 & 1800 \\
\hline
\end{tabular}

- Germicidal lamps (UVGI / UV-C) are mainly used in specialized sterilization processes. UV-C gives off light at the short end of the wavelength band that is harmful to microorganisms $253.7 \mathrm{~nm}$. UV-C lamps are used in air, water purification and some etching processes. Testing two of the ACs showed that no additional air cleaning was provided with the operation of an internal UV-C lamp; the internal UV-C lamps, however, inactivated $75 \%$ of fungal spores and $97 \%$ of bacteria cells captured in the air filter medium within 60 min (9). Research on UV air disinfection continues today. However, that evidence is accumulating, along with data on maintenance and safety in a wide variety of applications. It is now clearly understood, for example, that occupant motion and position within rooms greatly reduce the possibility of harmful overexposures to UV-C radiation in lower rooms. Interest and investment by major lighting fixture companies is badly needed to stimulate further development; however, the cost of applying upper-room UVGI is an important factor in poor countries, where UVGI is most critically needed to reduce transmission of TB, pandemic influenza, and other major airborne infectious threats (10).

- Studies demonstrate that UV sanitizers effectively kill bacteria, germs and microorganisms up to $99 \%$ in toothbrushes (430-450 nm). Many studies demonstrate that while they certainly reduce the amount of microscopic life, UV lights designed for toothbrushes seldom eliminate it entirely. UV light significantly decreased the number of bacteria compared to the control reduction in the number of $\mathrm{S}$. mutans (11). In another study, the results showed that after the sanitization procedure, there was a significant reduction $(\mathrm{P}<0.001)$ in microbial contamination in both microwave and UV group toothbrushes compared to control group toothbrushes, whereas the microbial count in the microwave group was significantly less compared to the UV group (12).

- UV exposure induces the production of vitamin D in the skin. A majority of positive health effects are related to this vitamin. It has regulatory roles in calcium metabolism (for normal functioning of the nervous system, for bone growth and bone density), immunity, cell proliferation, insulin secretion, and blood pressure. 
- Different proposed mechanisms causing a beneficial effect for the oral cavity include: 1.Vitamin D beneficially affects calcium metabolism, and 2 . Vitamin $D$ induces cathelicidin, an antimicrobial peptide, which attacks oral bacteria linked to dental caries. However, it is not proven that taking vitamin $\mathrm{D}$ will help treat dental caries or reduce the risk of developing dental caries. People with lower vitamin $\mathrm{D}$ levels had more attachment loss than those with higher levels.

- The sensitivity of oral bacteria to UV light was tested by subjecting plates streaked with bacteria to $254 \mathrm{~nm}$ UV. Direct exposure to relatively low doses of UV light $(2-7 \mathrm{~mJ} \mathrm{~cm} 2)$ effectively eliminated all bacterial strains tested. Furthermore, an Enterococcus faecalis strain, partially resistant to a $24 \mathrm{~h}$ exposure, was effectively eliminated within several seconds of exposure. UV was absorbed by a multilayer of bacteria. When 4 bacterial cells microm were present in the light path, the UV light dose had to be increased by a factor to achieve $100 \%$ elimination of the bacteria. The conclusion is that the application of UV light to eliminate endodontic pathogens may be possible. Nevertheless, its absorbance by outer layers of bacteria should be considered and the UV light dose adapted accordingly (13).

- UV-A and UV-C light induced hydrophilization of dental implants. The wettability of implant surfaces can be improved by UV irradiation. The efficiency of UV-A and UV-C irradiation to lower the CA by photocatalysis or photolysis, however, is strongly dependent on the specific material and surface. Thus, attempts to photofunctionalize these surfaces by irradiation is expected to result in a different pattern of bioresponses (14).

- The purpose of the study was to perform a preliminary in vitro test on the possible use of two different laser wavelengths, 405 and $532 \mathrm{~nm}$, to improve dental bleaching results. When hydrogen peroxide was present, only UVA irradiation was able to produce significant results, whereas when the photoFenton reaction occurred, all the three wavelengths used were able to produce a significant degradation of rhodamine $\mathrm{B}$. Within the limitations of this in vitro study, the light of the two laser devices, even remaining less effective than UV activation, showed its ability to improve the performance of bleaching agents based on Fenton photocatalysis (15).

\section{Conclusions}

- The application of UV in dentistry may have various effects and leads to benefits in different areas of dental practice. It is widely used both for diagnosis and therapy, which proves its significance.

\section{References}

1. Bush MA, Hermanson AS, Yetto RJ, et al. The use of ultraviolet LED illumination for composite resin removal: an in vitro study. Gen Dent. 2010 Sep-Oct; 58 (5): 214-218.

2. Hermanson AS, Bush MA, Miller RG, et al. Ultraviolet illumination as an adjunctive aid in dental inspection. J Forensic Sci. 2008 Mar; 53 (2): 408-411. 
3. Metzger Z, Better H, Abramovitz I. Immediate root canal disinfection with ultraviolet light: an ex vivo feasibility study. Oral Surg Oral Med Oral Pathol Oral Radiol Endod. 2007 Sep; 104 (3): 42533.

4. www.opticalaser.net/e-shop/product.php?ProductID=54

5. Mohammad T.AL -Khafagy, Israa K. AL Yasiri et al, Disinfection Of Alginate And Silicon Impressions By Using UV And Blue Light. (In Vivo Study), Journal of Kufa for Nursing Science Vol. (3) No. (2), 2013.

6. Panov Vl., Krasteva A., Disinfection of dental impression materials - review, Problems of dental medicine, XXXVI/2010 (2), 98-102.

7. Larsen T, Fiehn NE, Peutzfeldt A. et al.,Disinfection of dental impressions and occlusal records by ultraviolet radiation., Eur J Prosthodont Restor Dent. 2000 Jun; 8 (2): 71-74.

8. Gatti A. M., UV-radiation aging test on composite materials for dental restorations, Journal of Biomedical Materials Research 06/1987; 21 (5): 603-612.

9. Kujundzic E, Matalkah F, Howard CJ, et al. UV air cleaners and upper-room air ultraviolet germicidal irradiation for controlling airborne bacteria and fungal spores. J Occup Environ Hyg. 2006 Oct; 3 (10): 536-46.

10. Nicholas G. Reeda, The History of Ultraviolet Germicidal Irradiation for Air Disinfection, Public Health Rep. 2010 Jan-Feb; 125 (1): 15-27.

11. Bélanger-Giguère $K$, Giguère $S$, Bélanger $M$. Disinfection of toothbrushes contaminated with Streptococcus mutans. Am J Dent. 2011 Jun; 24 (3): 155-158.

12. Gujjari SK, Gujjari AK, Patel PV et al.Comparative evaluation of ultraviolet and microwave sanitization techniques for toothbrush decontamination. J Int Soc Prev Community Dent. 2011 Jan; 1 (1): 20-26.

13. Metzger Z1, Dotan M, Better H, Abramovitz I.Sensitivity of oral bacteria to $254 \mathrm{~nm}$ ultraviolet light. Int Endod J. 2007 Feb; 40 (2): 120-7.

14. Al Qahtani MS, Wu Y, Spintzyk Set al., UV-A and UV-C light induced hydrophilization of dental implants. Dent Mater. 2015 May 14. pii: S0109-5641 (15) 00131-1.

15. Lagori G1, Rocca J, Brulat N, et al. Comparison of two different laser wavelengths' dental bleaching results by photo-Fenton reaction: in vitro study, Lasers Med Sci. 2015 Apr; 30 (3): 1001-6. 


\title{
Corresponding author
}

\author{
Vladimir Panov \\ Department of Conservative Dentistry and Oral Pathology, Faculty of Dental Medicine, Medical \\ University-Varna \\ 84, Tzar Osvoboditel, 9000 Varna, Bulgaria \\ E-mail: vl_panov@abv.bg
}

